\title{
Numerical investigation of the jet formation through the oscillation of a bubble between a couple of parallel walls
}

\author{
R. M. B. Teymouri ${ }^{1} \&$ G. Ahmadi $^{2}$ \\ ${ }^{1}$ Center of Smart Interfaces, Darmstadt University of Technology, \\ Germany \\ ${ }^{2}$ Department of Mechanical and Aeronautical Engineering, \\ Clarkson University, USA
}

\begin{abstract}
The jet formation through the oscillation of a bubble between a couple of parallel rigid walls was numerically investigated. The Navier-Stokes and energy equations accompanying with the proper constitutive relations were numerically solved to predict the flow and thermal conditions inside and outside the bubble. The volume of fluid method was incorporated to capture the bubble surface applying a piecewise linear interface reconstruction method. For the bubble to walls distances less than certain limits, the bubble underwent a necking process after reaching to its maximum volume. The necking process led to splitting of the bubble and the formation of a couple of round jets toward the walls. The velocity of each jet was higher than the velocity of a jet induced through the oscillation of a bubble near a single wall. Also it was found that keeping the position of one of the walls fixed, the velocity of the jet toward that wall increases with increasing the other wall distance from the bubble, up to a certain limit. These analyses were performed for several initial inside to outside pressure ratios of the bubble. The results can be used for increasing the performance of the devices for which the jet induced through the near wall bubble oscillation is applied.
\end{abstract}

Keywords: oscillating bubble, wall, jet, VOF. 


\section{Introduction}

Bubble dynamics is one of the main subjects in the phenomenological studies of cavitation and boiling. Some of the discovered features have seemed to be useful for inventing new devices. One of the invented devices is the ink-jet printer. In this type of printers, the ink droplets are generated through the collapse of the boiling bubbles [1]. Another invented device is a micro-pump that does not use any moving element. In this pump, the jet induced through the near wall bubble oscillation is applied [2]. It should be reminded that this induced jet that leads to the bubble collapse, is the main mechanism of the cavitation damaging effects in the turbomachineries and hydraulic structures [3-5].

The jet formation through the near wall bubble oscillation is the subject of the present numerical study. To briefly describe the physics of this phenomenon, a high-pressure bubble is considered near a rigid wall. The bubble grows spherically to reach its maximum volume. During the expansion, the bubble potential energy that is related to its gas content, is transferred to the surrounding liquid in the form of kinetic energy. The momentum of the outgoing liquid however causes the bubble to over-expand. This energy transfer accompanies with the pressure decrease inside the bubble and results the deceleration of the expansion. At the end of the expansion, the pressure difference between inside and outside the bubble has an opposite sign with respect to the start of this process and leads to initiation of the compression. The compression is also a result of the surface tension. During the compression, the surrounding liquid kinetic energy is transferred to the bubble gas content in the form of potential energy, but with a lower amount as a result of the viscous damping effects. The main effect of the wall is that the surrounding liquid kinetic energy in the regions away from the wall is much higher than the region adjacent to the wall. Consequently a part of the ingoing liquid penetrates into the bubble as a high velocity round jet toward the wall. The jet destroys the bubble spherical shape and makes a hole and finally impacts the wall. Sequence of the jet formation through the oscillation of a bubble near a wall is shown in Fig. 1.

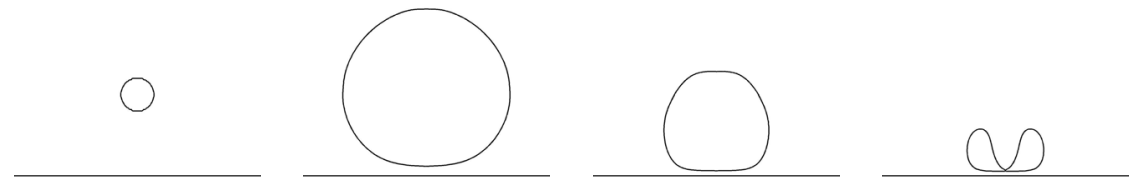

Figure 1: Jet formation through the oscillation of a bubble near a wall.

Lauterborn and Bolle [6] carried out several experiments on the near wall bubble oscillation and observed the instantaneous bubble shapes using the highspeed photography. The method for generating the bubble was firing a focused short laser pulse. It should be pointed that one of the problems in performing such experiments is the lack of possibility to measure the initial pressure inside the bubble as well as the equilibrium bubble radius. Consequently, for the 
numerical simulations one is confronted with making assumptions on the initial conditions.

In the experiments carried out by Ishida et al. [7] the jet formation through the oscillation of a bubble between a couple of parallel walls was investigated. The bubble was generated by an electric charge. According to their observations, when the distances between the bubble and the walls are less than a certain limit, the bubble undergoes a necking process during the compression. The necking process leads to splitting of the bubble into a couple of compressing daughter bubbles. In turn, a couple of jets toward the walls are induced. They found that keeping one of the walls position fixed, the velocity of the jet toward that wall increases with increasing the other wall distance from the bubble.

The necking process is the result of the fact that the surrounding liquid kinetic energy in the regions away from the walls is much higher than the regions adjacent to the walls. Consequently a part of the ingoing liquid penetrates into the bubble as a plane jet parallel to the walls, leading to necking of the bubble. At the moment of the bubble splitting, a stagnation point is created at the splitting point. Therefore the plane jet is converted to a couple of round jets toward the walls. The round jets intensify the jets induced during the compression of the daughter bubbles. Consequently, the jets impact the walls with the velocities higher than the velocity of the jet induced through the oscillation of a bubble near a single wall. Sequence of the jet formation through the oscillation of a bubble between a couple of parallel walls is shown in Fig. 2.
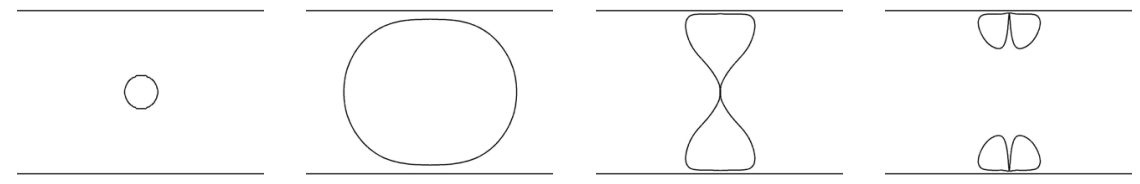

Figure 2: The jet formation through the oscillation of a bubble between a couple of parallel walls.

The numerical studies about the jet formation through the near wall bubble oscillation can be divided into two categories. At the first category, the fluid flow is assumed to be inviscide and irrotational. To calculate the flow field variables, a Laplace equation governing the velocity potential is solved [1]. This approach is simple for implementation. But the inviscide fluid assumption is no longer acceptable in the small scales [5]. The irrotational flow assumption is also incorrect because of the vortical flow structure inside the bubble during the jet formation. In the second category, Navier-Stokes equations are solved accompanying with the different methods to capture the bubble surface [5].

In the present study the second approach is selected to model the jet formation through the oscillation of a bubble between a couple of parallel walls. The simulations are performed for several initial inside to outside pressure ratios of the bubble. One of the walls position is kept fixed and the effects of changing the other wall position are investigated. 


\section{Mathematical modelling}

The fluid flow field under consideration contains two immiscible phases with a sharp interface across which, no mass transfer occurs. The primary phase is compressible air inside the bubble and the secondary phase is incompressible water outside the bubble.

\subsection{Interface capturing approaches}

The recent numerical methods developed to precisely capture the sharp interfaces between the immiscible fluids on Eulerian grids, can be divided into a couple of approaches called the surface approach and the volume approach.

In the surface approach, the interface is represented by the marker points. The most important advantage of this approach is that the interface remains sharp as it is advected across the domain. This results precisely calculation of the interface curvature. There are a couple of mostly used methods that can be categorized in this approach, including the front tracking method and the level set method. In the front tracking method the interface is represented by a set of connected massless marker particles and explicitly tracked in a Lagrangian manner using the flow field local velocity. By using this method, some difficulties arise in modelling the coalescence or break up of the interfaces. In the level set method a scalar advection equation is solved for a distance function from the interface. The interface is defined as the zero level set of the distance function and implicitly captured during its advection across the domain. The disadvantage of the level set method is that there is no guaranty for the mass conservation during the interface advection [8].

In the volume approach, the immiscible fluids on either sides of the interface are marked instead of marking the interface itself. Consequently some special techniques are needed to reconstruct the sharp interface. There are a couple of mostly used methods that can be categorized in this approach, including the marker and cell method (MAC) and the volume of fluid method (VOF). In the MAC method the marker particles that are used to mark the fluids, are transported in a Lagrangian manner as the interface is advected across the domain. The interface is reconstructed using the marker particle density in the mixed numerical cells where the marker particles of both of the fluids exist. The disadvantage of this method is the computational cost due to the requirement of many particles. In the VOF method an indicator function that is the local volume fraction of one of the fluids, is used to distinguish between two different fluids. The indicator function is locally calculated solving a scalar equation. The interface is reconstructed using the indicator function in the mixed cells where its values are in the range of zero to one. The disadvantage of this method is the difficulties to precisely calculate the interface local curvature [8].

\subsection{VOF method}

For the present research the VOF method is used to numerically capture the bubble surface. In this method an indicator function that is the local volume 
fraction of one of the fluids, is initialized in each cell. As a result of the fact that the fluids are immiscible, each fluid element does not change its material in time. Therefore the indicator function satisfies eqn (1) that is called the volume fraction equation [9]. In the present study this equation is written in term of the volume fraction of water.

$$
\frac{D}{D t}\left(\alpha_{\text {water }}\right)=\left(\rho_{\text {water }} \alpha_{\text {water }}\right)_{t}+\left(\rho_{\text {water }} \alpha_{\text {water }} v_{i}\right)_{, i}=0
$$

where $\rho$ is the density, $\alpha$ is the volume fraction and $v$ is the velocity. In this paper the equations are written in the tensor form.

Solving eqn (1) using the lower order schemes, leads to losing the interface sharpness due to the numerical diffusion. Using the higher order schemes is also not suitable because they make the solution unstable. Several techniques have been proposed to precisely predict a sharp interface using the VOF method. One of the accurate techniques is to represent the interface by a piecewise linear surface. In this geometrical technique the interface in each cell is represented by a plane in $3 \mathrm{D}$ or a line in $2 \mathrm{D}$ that is perpendicular to the local gradient of the volume fraction. At the present study, Young's method is used to calculate the local gradient of the volume fraction. After calculating the orientation of the linear surface in each cell, its position can be obtained by knowing the volume fractions of both of the fluids.

\subsection{Governing equations}

In the VOF method the fluid flow equations are written in terms of the mixture properties to make a single set of the governing equations for both of the fluids. The mixture properties can be calculated using eqn (2) that is written in terms of a general fluid property $\varphi$.

$$
\varphi_{\text {mix }}=\alpha_{\text {air }} \varphi_{\text {air }}+\alpha_{\text {water }} \varphi_{\text {water }}
$$

Equation (3) is the continuity equation.

$$
\left(\rho_{\text {mix }}\right)_{t}+\left(\rho_{\text {mix }} v_{i}\right)_{, i}=0
$$

Equation (4) is the momentum equation.

$$
\left(\rho_{m i x} v_{j}\right)_{t}+\left(\rho_{m i x} v_{j}\right)_{, i} v_{i}+p_{, j}-\tau_{i j, i}-F_{j}=0
$$

where $p$ is the pressure, $F$ is the body force due to the surface tension and $\tau$ is the viscous stress. Equation (5) is used to calculate the viscous stress tensor.

$$
\tau_{i j}=\mu_{m i x}\left(v_{i j}+v_{j i}\right)
$$

where $\mu$ is the viscosity. 
At the present study, the total energy and temperature are treated as massaveraged variables. The mass-averaged variables are calculated using eqn (6) that is written in term of a general variable $\mathrm{X}[10]$.

$$
\mathrm{X}=\frac{\alpha_{\text {air }} \rho_{\text {air }} \mathrm{X}_{\text {air }}+\alpha_{\text {water }} \rho_{\text {water }} \mathrm{X}_{\text {air }}}{\alpha_{\text {air }} \rho_{\text {air }}+\alpha_{\text {water }} \rho_{\text {water }}}
$$

Equation (7) is the energy equation.

$$
\left(\rho_{m i x} E\right)_{t}+\left(v_{i}\left(\rho_{m i x} E+p\right)\right)_{, i}=\left(k_{m i x} T_{, i}\right)_{, i}
$$

where $E$ is the total energy, $T$ is the temperature and $k$ is the thermal conductivity.

The total energy and temperature fields are related together using eqn (8).

$$
E=c_{P_{-} \text {mix }} T-\frac{p}{\rho_{\text {mix }}}+\frac{1}{2} v_{i} v_{i}
$$

where $c_{P}$ is the constant pressure specific heat.

The ideal gas equation is used to calculate the variable air density.

\subsection{Surface tension}

A continuum surface force model (CSF) is used to take the surface tension effects into account [11]. The surface tension is written in term of the pressure jump across the interface. The pressure jump is related to the interface curvature that is the divergence of the interface unit normal. Applying the divergence theorem, the surface tension can be expressed as a volume force. This volume force that can be calculated using eqn (9) is added to the momentum equation as a source term.

$$
F=\sigma \frac{\rho_{m} \kappa \alpha_{\text {water }, i}}{0.5\left(\rho_{\text {air }}+\rho_{\text {water }}\right)}
$$

where $\sigma$ is the surface tension and $\kappa$ is the interface curvature.

\subsection{Numerical schemes}

FLUENT CFD software is used to numerically solve the governing equations applying the finite volume method. SIMPLE pressure based algorithm is applied to solve the set of governing equations. A first order implicit time integration method is used for the temporal discretization. The power law first order scheme is used for discretization of the convective terms.

According to the axisymmetry of the flow field, the equations are written in a cylindrical coordinate system. The line that is perpendicular to the walls and passes the bubble center is chosen as the axis of the cylindrical coordinate. 
Because of the symmetry with respect to this axis, the equations are solved in a plane passing the axis. Therefore the solution domain is simply a rectangle.

The upper and lower sides of the rectangular solution domain are the walls for which, the no-slip boundary condition is imposed. The axis boundary condition is imposed on the right side of the domain and a constant static pressure is imposed on the left side. The constant static pressure boundary is sufficiently away from the axis. Therefore the variable pressure field around the oscillating bubble is not affected by the constant pressure boundary.

GAMBIT grid generation software is used to generate the two-dimensional structured numerical grids. The numbers of the cells used for the different cases are in the range of 8500 to 15000 . The numerical grids are examined to result grid independent solutions.

\section{Problem description}

A bubble with the initial diameter of $1 \mathrm{~mm}$ is considered between a couple of parallel rigid walls that are large in comparison with the bubble size. Therefore the flow field is axisymmetric with respect to an axis that is perpendicular to the walls and passes the bubble center. For the different cases, the values of 20, 40, 60, 80 and 100 are assigned for the initial inside to outside pressure ratio of the bubble. This parameter is represented by $\left(\mathrm{p}_{\text {in }} / \mathrm{p}_{\text {out }}\right)_{\text {initial }}$. For each value of $\left(p_{\text {in }} / p_{\text {out }}\right)_{\text {initial }}$, the distance from the bubble center to each wall is nondimensionalized with the maximum bubble radius. The non-dimensional distances from the bubble center to the lower and upper walls are represented by $\gamma_{1}$ and $\gamma_{2}$ respectively. The value of $\gamma_{1}$ is kept 1 for all of the cases. The values of $\gamma_{2}$ are in the range of 1 to 2.5 for the different cases.

To find the bubble maximum radius, a free bubble oscillation is simulated for each value of $\left(\mathrm{p}_{\mathrm{in}} / \mathrm{p}_{\text {out }}\right)_{\text {initial }}$. For the initialization of the numerical solution, the initial temperature inside the bubble should be calculated. Assuming an isentropic process between the initial and equilibrium conditions, eqns (10) and (11) can be used:

$$
\begin{aligned}
& \frac{p_{\text {in,initial }}}{p_{\text {in,equilibrium }}}=\left(\frac{R_{\text {equilibrium }}}{R_{\text {initial }}}\right)^{3 k} \\
& \frac{T_{\text {in,initial }}}{T_{\text {in,equilibrium }}}=\left(\frac{R_{\text {equilibrium }}}{R_{\text {initial }}}\right)^{3(k-1)}
\end{aligned}
$$

where $R$ is the bubble radius and $k$ is a constant value that is 1.4 for the present study.

Equation (12) is obtained by applying a force balance at the equilibrium condition.

$$
p_{\text {in,equilibrium }}-p_{\text {out }}=\frac{2 \sigma}{R_{\text {equilibrium }}}
$$


Eliminating $p_{\text {in,equilibrium }}$ in relating eqns (10) and (12), the equilibrium bubble radius can be obtained for the different values of $\left(\mathrm{p}_{\text {in }} / \mathrm{p}_{\text {out }}\right)_{\text {initial. }}$. Assuming that the inside and outside temperatures of the bubble have the same values at the equilibrium condition, eqn (11) can be used to find the temperature inside the bubble at the initial condition.

To indicate the importance of the buoyancy force in analyzing the bubble oscillation, a non-dimensional parameter is usually used in the literature. This parameter that is represented by $\Delta$ is defined by eqn (13).

$$
\Delta^{2}=\frac{\rho_{\text {water }} g R_{\max }}{p_{\text {out }}-p_{V}}
$$

where $g$ is the gravity acceleration and $p_{\mathrm{V}}$ is the vapor pressure. The effects of the buoyancy force are negligible as long as the value of $\Delta$ is less than 0.3 [12]. For the present study the effects of buoyancy force are negligible.

\section{$4 \quad$ Numerical results}

Figure 3 shows the numerical simulation of a bubble oscillation between a couple of parallel walls. The initial inside to outside pressure ratio of the bubble is 100 and the non-dimensional distances from the bubble center to both of the walls are 1. The necking process, splitting and formation of the round jets toward the walls are successfully simulated. The instantaneous bubble shapes are qualitatively in good agreement with the experimental observations performed by Ishida et al. [7]. The numerical and experimental results cannot be
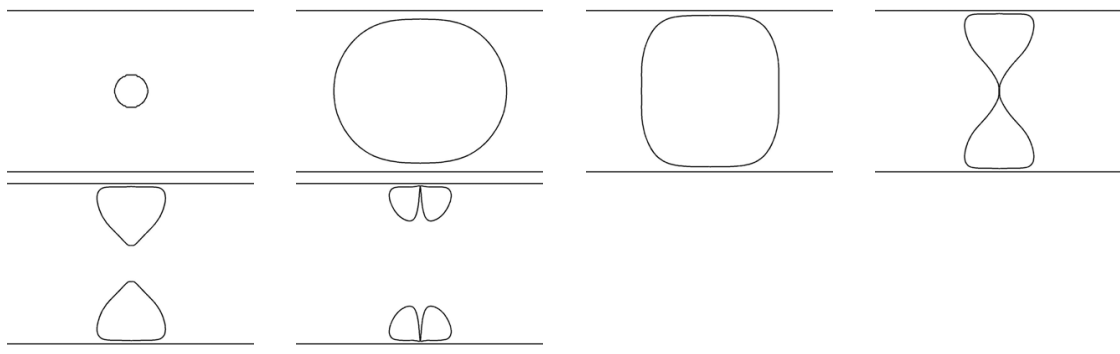

Figure 3: Simulation of a bubble oscillation between a couple of parallel walls, $\left(\mathrm{p}_{\text {in }} / \mathrm{p}_{\text {out }}\right)_{\text {initial }}=100, \gamma_{1,2}=1$.
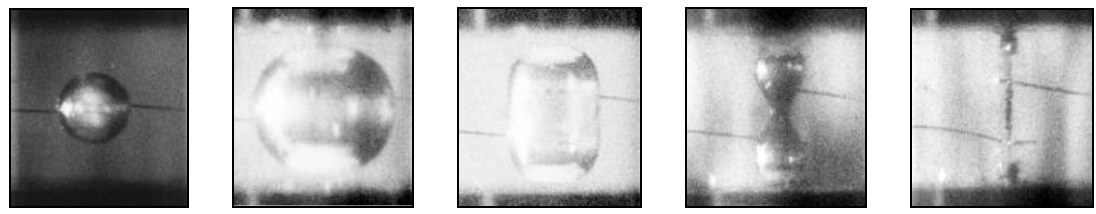

Figure 4: Experimental observation of a bubble oscillation between a couple of parallel walls, $\gamma_{1,2}=1.1$, [7]. 
quantitatively compared, because neither the initial pressure inside the bubble is possible to be measured nor the equilibrium bubble radius.

Figure 4 shows the experimental observations carried out by Ishida et al. In the experiment, the non-dimensional distances of the bubble center to both of the walls are 1.1 .

Figures 5 and 6 show the velocity vector field obtained by the numerical simulation of the case for which $\left(\mathrm{p}_{\mathrm{in}} / \mathrm{p}_{\text {out }}\right)_{\text {initial }}$ is 100 and both of $\gamma_{1}$ and $\gamma_{2}$ are 1 . Figure 5 demonstrates the creation of a stagnation point at the point of splitting and the formation of the round jets toward the walls. Figure 6 shows the velocity vector field around one of the daughter bubbles at the moment of the creation of a hole by the round jet.

Figure 7 shows the bubble shapes at the moment of splitting, obtained by the numerical simulation of the different cases. For each value of $\gamma_{2}$, the different bubble shapes corresponding to the different values of $\left(\mathrm{p}_{\mathrm{in}} / \mathrm{p}_{\text {out }}\right)_{\text {initial }}$, are shown in a single picture. The bigger bubble shapes correspond to the higher values of $\left(p_{\text {in }} / p_{\text {out }}\right)_{\text {initial. }}$ In this figure the bubble shapes are shown just for the values of $\gamma_{2}$ which are in the range of 1 to 1.5 , because the splitting process does not occur for the higher values. For the value of $\gamma_{2}$ equal to 1.5 , the splitting process does not occur if the value of $\left(\mathrm{p}_{\text {in }} / \mathrm{p}_{\text {out }}\right)_{\text {initial }}$ is higher than 20 . It is also demonstrated from fig. 7 that for the higher values of $\gamma_{2}$, the upper daughter bubble has the smaller sizes, but the size of the lower daughter bubble remains fixed. This behaviour is consistent with the other experimental observations carried out by Ishida et al. [7]. In that experiment, the value of $\gamma_{1}$ is 1.3 and the value of $\gamma_{2}$ is 0.9. The experimental observations are shown in fig. 8.

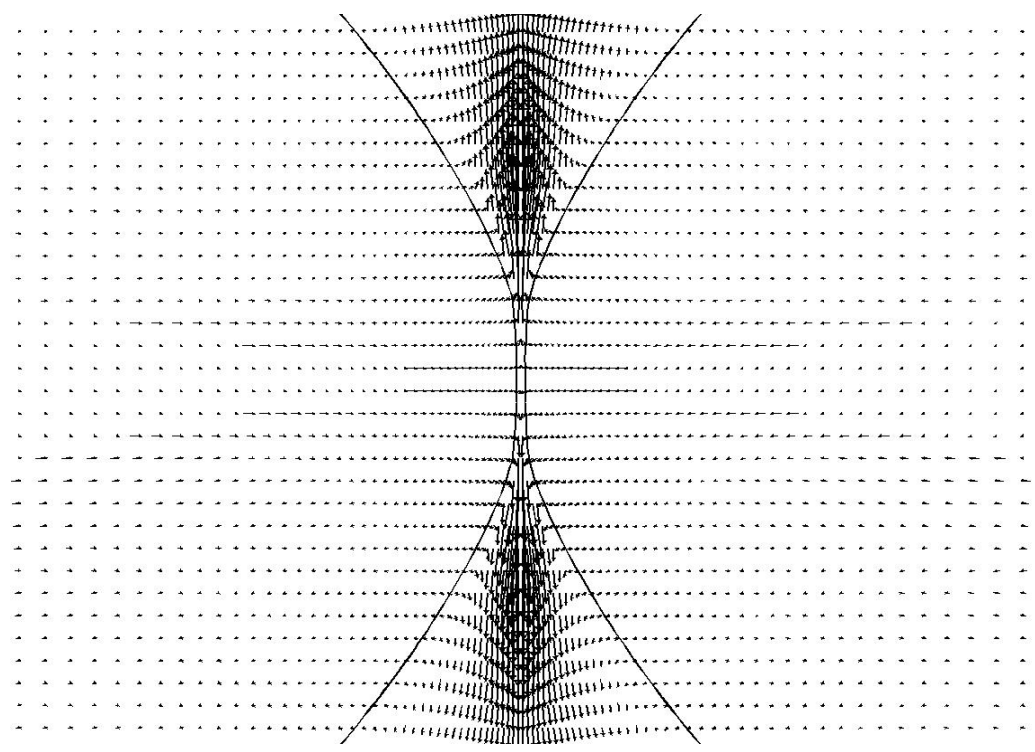

Figure 5: Velocity vector field at the moment of splitting, $\left(\mathrm{p}_{\text {in }} / \mathrm{p}_{\text {out }}\right)_{\text {initial }}=100$, $\gamma_{1,2}=1$. 


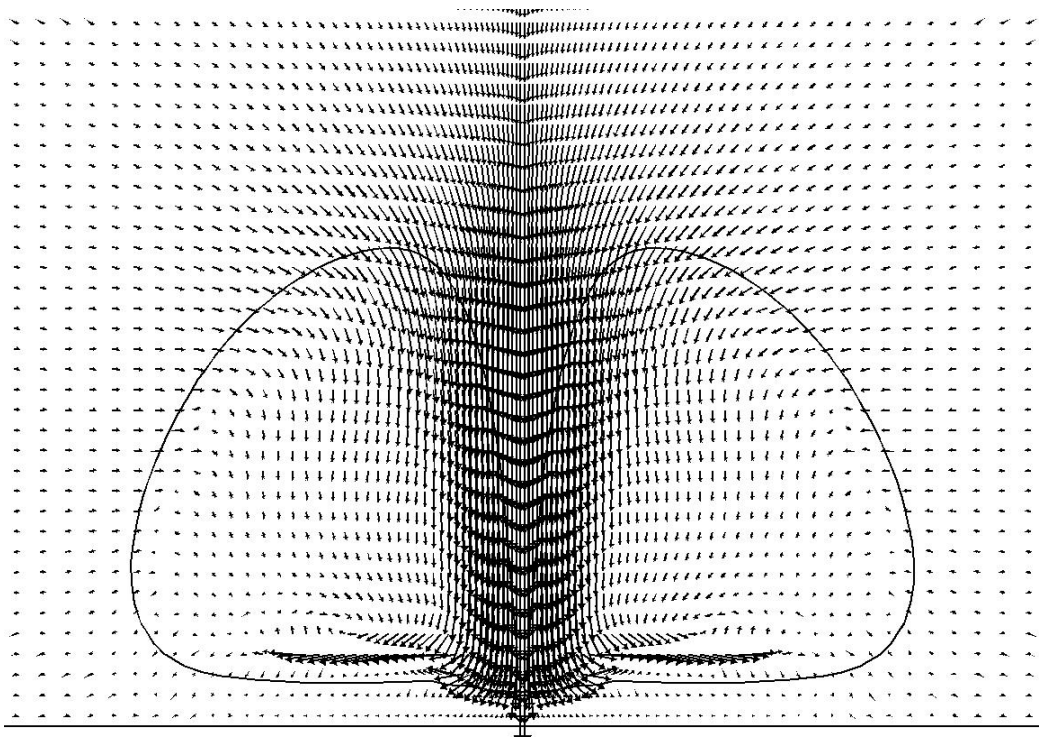

Figure 6: Velocity vector field at the moment of the creation of a hole, $\left(\mathrm{p}_{\text {in }} / \mathrm{p}_{\text {out }}\right)_{\text {initial }}=100, \gamma_{1,2}=1$.

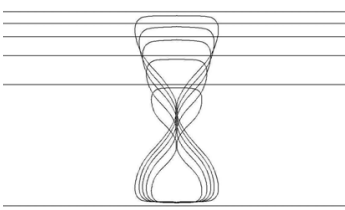

$\gamma_{2}=1$

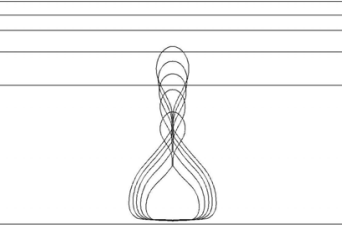

$\gamma_{2}=1.3$

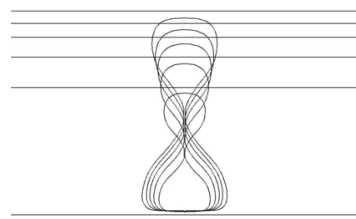

$\gamma_{2}=1.1$

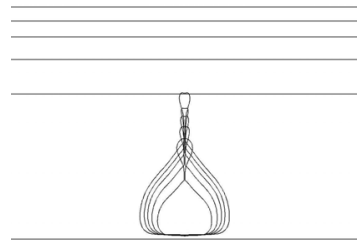

$\gamma_{2}=1.4$

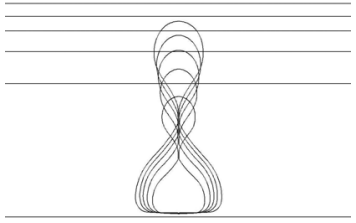

$\gamma_{2}=1.2$

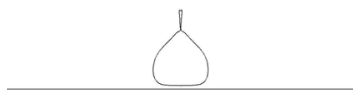

$\gamma_{2}=1.5$

Figure 7: The bubble shapes at the moment of splitting for the different cases.
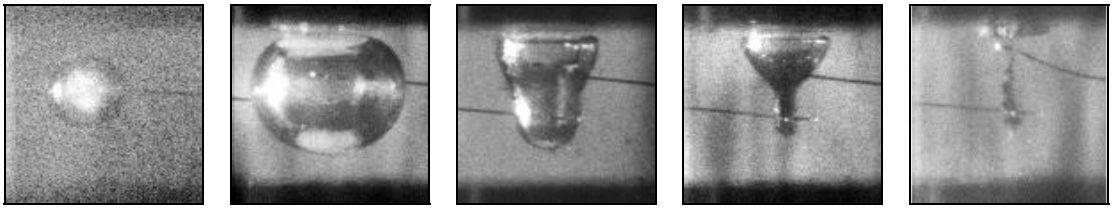

Figure 8: Experimental observation of a bubble oscillation between a couple of parallel walls, $\gamma_{1}=1.3$ and $\gamma_{2}=0.9$, [7]. 

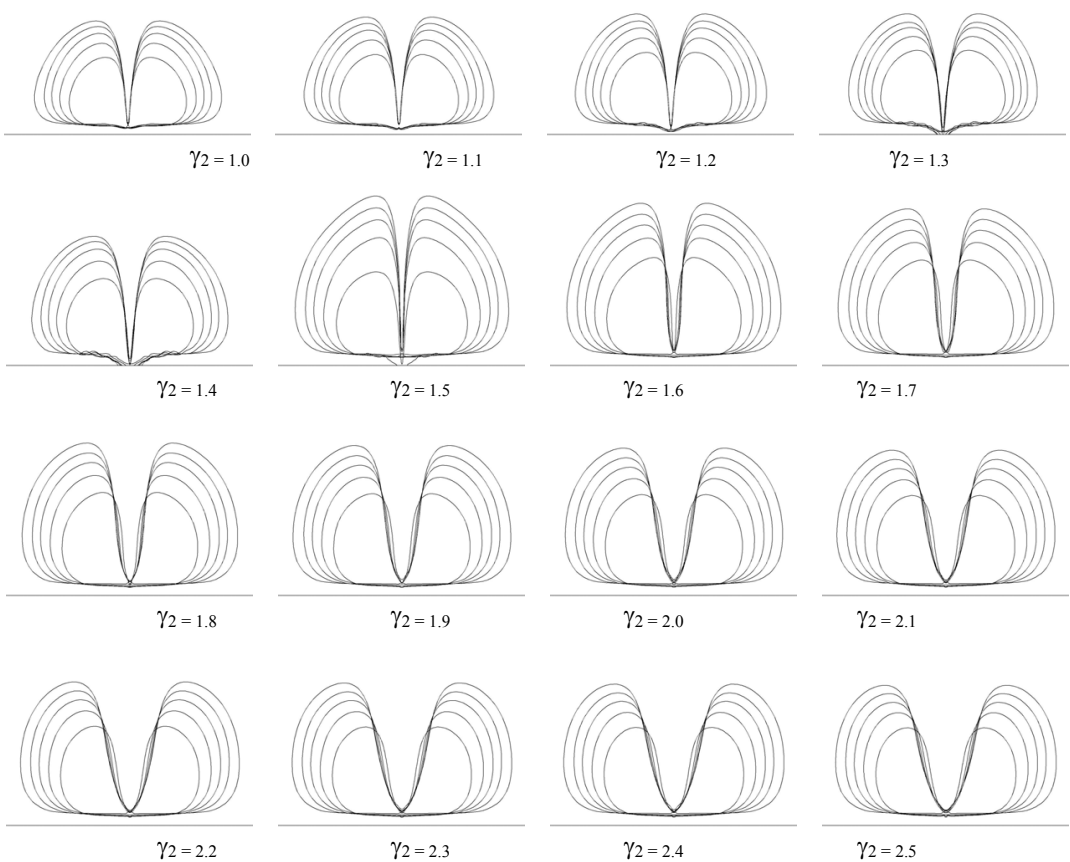

Figure 9: Bubble shapes at the moment of creation of a hole for the different cases.

Figure 9 shows the bubble shapes near the lower wall at the moment of creation of a hole. The results are obtained by the numerical simulation of the different cases. For each value of $\gamma_{2}$, the different bubble shapes corresponding to the different values of $\left(\mathrm{p}_{\text {in }} / \mathrm{p}_{\text {out }}\right)_{\text {initial }}$, are shown in a single picture. The bigger bubble shapes correspond to the higher values of $\left(\mathrm{p}_{\text {in }} / \mathrm{p}_{\text {out }}\right)_{\text {initial. }}$. It is demonstrated from this figure that as the value of $\gamma_{2}$ increases, the diameter of the induced round jet toward the lower wall decreases. Also for the cases without splitting, the bubble near the lower wall has bigger sizes than the cases with splitting.

Figure 10 shows the effect of the value of $\left(\mathrm{p}_{\text {in }} / \mathrm{p}_{\text {out }}\right)_{\text {initial }}$ on the jet velocity for the cases for which $\gamma_{2}$ tends to infinity. The velocities are measured at the moment of the creation of a hole. The jet velocity of the case for which the value of $\left(\mathrm{p}_{\text {in }} / \mathrm{p}_{\text {out }}\right)_{\text {initial }}$ is 20 , is used to non-dimensionalize the velocities of the other cases. This figure demonstrates that increasing the value of $\left(\mathrm{p}_{\text {in }} / \mathrm{p}_{\text {out }}\right)_{\text {initial }}$ leads to increasing the jet velocity.

Figure 11 shows the effect of the values of $\gamma_{2}$ and $\left(p_{\text {in }} / p_{\text {out }}\right)_{\text {initial }}$ on the jet velocity toward the lower wall at the moment of the creation of a hole. For each value of $\left(\mathrm{p}_{\mathrm{in}} / \mathrm{p}_{\text {out }}\right)_{\text {initial }}$, the jet velocities are non-dimensionalized with the jet velocity of the case for which $\gamma_{2}$ tends to infinity. All of the non-dimensional velocities have the values higher than 1 . Therefore it is demonstrated from this figure that the upper wall increases the jet velocity toward the lower wall. For 
each value of $\gamma_{2}$, the higher values of $\left(\mathrm{p}_{\text {in }} / \mathrm{p}_{\text {out }}\right)_{\text {initial }}$ result the higher increases in the jet velocity, because the kinetic energy of the surrounding liquid is higher for the higher pressure ratios. For each value of $\left(\mathrm{p}_{\text {in }} / \mathrm{p}_{\text {out }}\right)_{\text {initial }}$, the jet velocity toward the lower wall increases with increasing the value of $\gamma_{2}$ up to a certain limit. It can be a result of the fact that for the values of $\gamma_{2}$ higher than 1 , most of the plane jet kinetic energy is transferred to the round jet induced toward the lower wall. For the value of $\left(\mathrm{p}_{\text {in }} / \mathrm{p}_{\text {out }}\right)_{\text {initial }}$ equal to 20 , this limit is 1.6. But for the higher values of $\left(p_{\text {in }} / p_{\text {out }}\right)_{\text {initial }}$ this limit is 1.5 . One of the features of the cases for which the value of $\left(\mathrm{p}_{\text {in }} / \mathrm{p}_{\text {out }}\right)_{\text {initial }}$ is 20 , is that the splitting process occurs also at the value of $\gamma_{2}$ equal to 1.5 . Therefore it can be concluded that as long as the splitting process occurs, the jet velocity toward the lower wall increases with increasing the value of $\gamma_{2}$. To interpret this result, it can be stated that for the cases without the splitting process, the induced plane jet is weak and can not sufficiently intensify the induced round jet during the bubble compression.

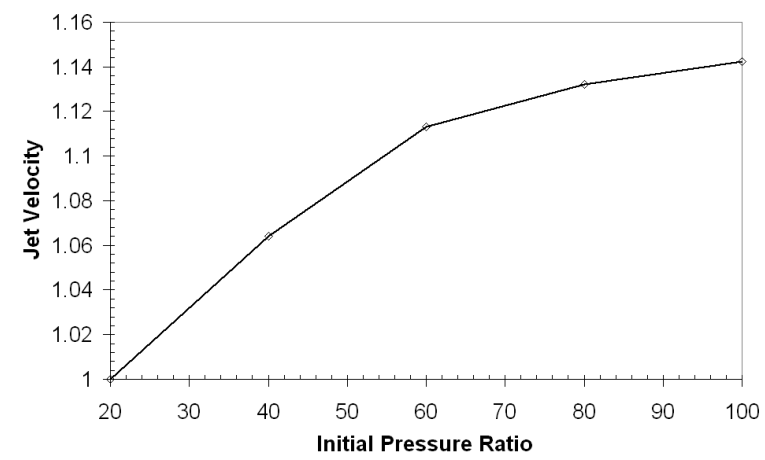

Figure 10: The jet velocity versus the value of $\left(\mathrm{p}_{\text {in }} / \mathrm{p}_{\text {out }}\right)_{\text {initial }}$ at the moment of the creation of a hole for the cases for which $\gamma_{2}$ tends to infinity.

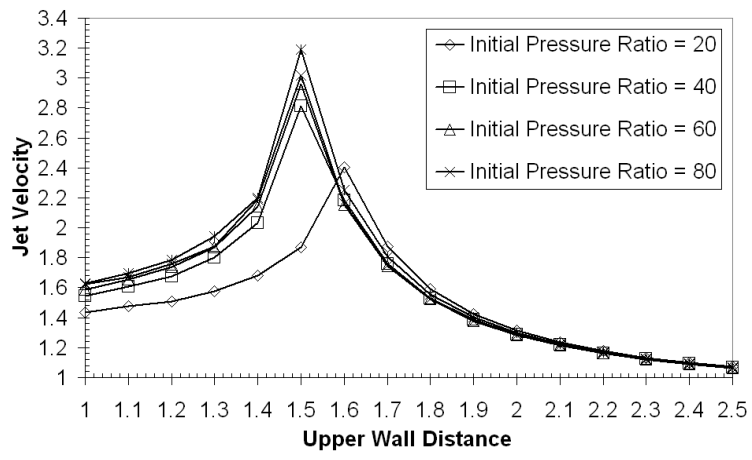

Figure 11: The jet velocity versus the value of $\gamma_{2}$ at the moment of the creation of a hole for the different values of $\left(\mathrm{p}_{\text {in }} / \mathrm{p}_{\text {out }}\right)_{\text {initial. }}$. 


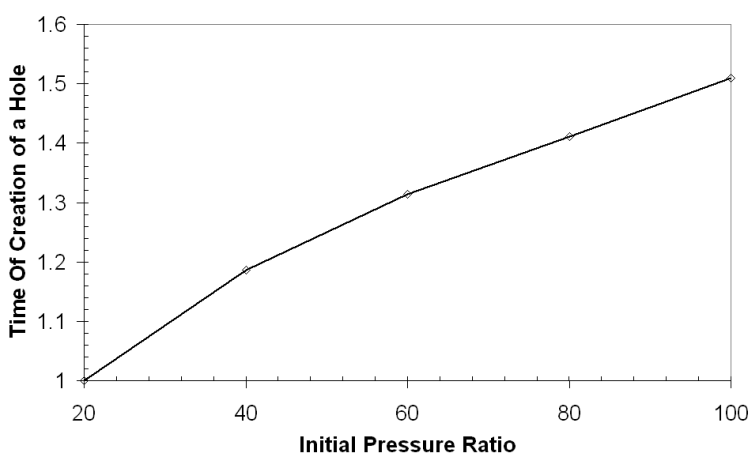

Figure 12: The time of the creation of a hole versus $\left(\mathrm{p}_{\text {in }} / \mathrm{p}_{\text {out }}\right)_{\text {initial }}$ for the cases for which $\gamma_{2}$ tends to infinity.

Figure 12 shows the effect of the value of $\left(\mathrm{p}_{\text {in }} / \mathrm{p}_{\text {out }}\right)_{\text {initial }}$ on the time of the creation of a hole, for the cases for which $\gamma_{2}$ tends to infinity. In this figure, the times are non-dimensionalized with the time of the creation of a hole for the case for which the value of $\left(\mathrm{p}_{\mathrm{in}} / \mathrm{p}_{\text {out }}\right)_{\text {initial }}$ is 20 . It is demonstrated from this figure that for the higher values of $\left(\mathrm{p}_{\mathrm{in}} / \mathrm{p}_{\text {out }}\right)_{\text {initial }}$ the process that leads to the creation of a hole takes more time.

Figure 13 shows the effects of $\left(p_{\text {in }} / p_{\text {out }}\right)_{\text {initial }}$ and $\gamma_{2}$ on the time of the creation of a hole. For each value of $\left(\mathrm{p}_{\mathrm{in}} / \mathrm{p}_{\text {out }}\right)_{\text {initial, }}$, the times are nondimensionalized with the time of the creation of a hole for the case for which $\gamma_{2}$ tends to infinity. Figure 13 demonstrates that for each value of $\left(p_{\text {in }} / p_{\text {out }}\right)_{\text {initial }}$, the process that leads to the creation of a hole takes more time for the lower values of $\gamma_{2}$.

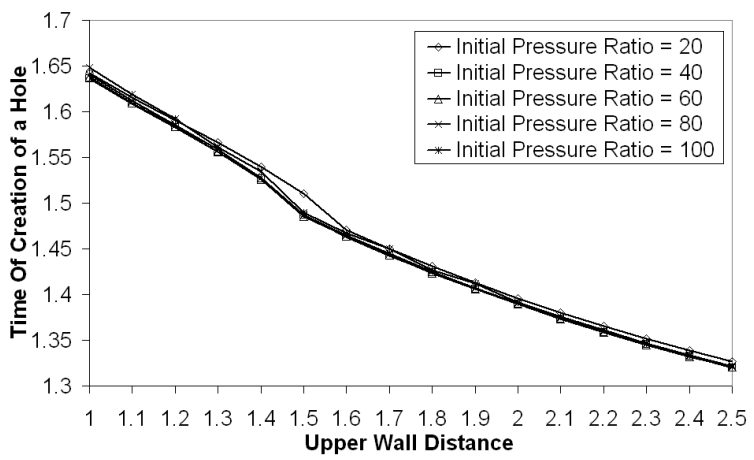

Figure 13: The time of the creation of a hole versus the value of $\gamma_{2}$ for the different values of $\left(\mathrm{p}_{\text {in }} / \mathrm{p}_{\text {out }}\right)_{\text {initial. }}$. 


\section{Conclusion}

The jet formation through the oscillation of a bubble between a couple of parallel walls is numerically simulated using FLUENT CFD software. The volume of fluid method is used to capture the bubble surface applying a geometric reconstruction method. The simulations are performed for several initial inside to outside pressure ratios of the bubble and different distances from the walls to the bubble center. The results are qualitatively in good agreement with the experimental observations performed by Ishida et al. [7]. It is concluded that the existence of each wall increases the velocity of the round jet induced toward the other wall. Keeping the position of one of the walls fixed, the velocity of the jet toward that wall increases with increasing the other wall distance from the bubble center up to a certain limit. For the distances larger than this limit, the bubble splitting does not occur. Also it can be concluded that for the smaller distances from the walls to the bubble center, the process that leads to the jet formation takes more time.

\section{References}

[1] Wikipedia Free Encyclopedia, http://en.wikipedia.org/wiki/Inkjet_printer

[2] Khoo, B. C., Klaseboer, E. and Hung, K. C., A collapsing bubble-induced micro-pump using the jetting effect, J. Sensors and Actuators A, 118, pp. 152-161, 2005

[3] Brennen, C. E., Cavitation and bubble dynamics, Book, Oxford Univ. Press, 1995

[4] Benjamin, T. B. and Ellis A. T., The collapse of cavitation bubbles and pressure thereby produced against solid boundaries, Phil. Trans. Royal Soc. London A, 260, pp. 221-240, 1966

[5] Popinet, S. and Zaleski, S., Bubble collapse near a solid boundary: A numerical study of the influence of viscosity, J. Fluid Mech., 464, pp. 137163,2002

[6] Lauterborn, W. and Bolle, H., Experimental investigation of cavitationbubble collapse in the neighborhood of a solid boundary, J. Fluid Mech., 72, pp. 391-399, 1975

[7] Ishida, H., Nuntadusit, C., Kimoto, H., Nakagava, T. and Yamamoto, T., Cavitation bubble behavior near solid boundaries, CAV2001 Conf., 2001

[8] Gopala, V. R., van Wachen, B. G. M., Volume of fluid methods for immiscible-fluid and free-surface flows, Chem. Eng. J., 141, pp. 204-221, 2008

[9] Aulisa, E., Manservisi, S., Scardovelli, R., Zaleski, S., Interface reconstruction with least-squares fit and split advection in threedimensional Cartesian geometry, J. Comp. Phys., 225, pp. 2301-2319, 2007

[10] FLUENT 6.3 documentation

[11] Brackbill, J. U. and Kothe, D. B. and Zemach, C., A continuum method for modeling surface tension, J. Comp. Phys., 100, pp. 335-354, 1992

[12] Brujan, E. A., Pearson, A., Blake, J. R., Pulsating, buoyant bubbles close to a rigid boundary and near the null final Kelvin impulse state, Int. J. Multiphase Flow, 31, pp. 302-317, 2005 